\title{
LUPUS SCIENCE\& MEDICINE \\ Interferon pathway in SLE: one key to unlocking the mystery of the disease
}

\author{
Lars Rönnblom, ${ }^{\oplus}$ Dag Leonard
}

To cite: Rönnblom L, Leonard D. Interferon pathway in SLE: one key to unlocking the mystery of the disease. Lupus Science \& Medicine 2019;6:e000270. doi:10.1136/ lupus-2018-000270

Received 18 June 2019 Revised 19 July 2019 Accepted 30 July 2019
Check for updates

(C) Author(s) (or their employer(s)) 2019. Re-use permitted under CC BY-NC. No commercial re-use. See rights and permissions. Published by BMJ.

Department of Medical Sciences, Rheumatology, Uppsala University, Uppsala, Sweden

Correspondence to Professor Lars Rönnblom; lars. ronnblom@medsci.uu.se

\section{ABSTRACT}

SLE is characterised by an activation of the interferon (IFN) system, which leads to an increased expression of IFN-regulated genes. The reasons behind the IFN signature in SLE are (1) the existence of endogenous IFN inducers, (2) activation of several IFN-producing cell types, (3) production of many different IFNs, (4) a genetic setup promoting IFN production and (5) deficient negative feedback mechanisms. The consequences for the immune system is a continuous stimulation to an immune response, and for the patient a number of different organ manifestations leading to typical symptoms for SLE. In the current review, we will present the existing knowledge of the IFN system and pathway activation in SLE. We will also discuss how this information can contribute to our understanding of both the aetiopathogenesis and some organ manifestations of the disease. We will put forward some issues that are unresolved and should be clarified in order to make a proper stratification of patients with SLE, which seems important when selecting a therapy aiming to downregulate the IFN system.

\section{INTRODUCTION}

SLE has for many years been a challenge to clinicians and a mystery for the basic scientist because of the complex clinical picture and the bewildering array of different aberrations in the immune system. Gradually, a number of observations in several research groups have unravelled important mechanisms behind the many clinical and laboratory findings, which now are translated into new therapies. However, several clinical trials have failed and there are a number of reasons for this. One cause is the fact that we still do not know how the genetic setup, environmental factors and stochastic events contribute to the initiation of the disease and the continuous autoimmune process. Obviously, several key elements of SLE need to be understood in more detail in order to completely unlock the secret behind the disease. Among key findings in SLE is a prominent expression of interferon (IFN)-regulated genes, an IFN signature, in blood and tissues. ${ }^{1-4}$ This observation was reported by several groups already in 2003 and initiated an intense activity among researchers trying to explain the finding. Simultaneously, colleagues started to investigate if the IFN signature could be linked to clinical phenotype, disease activity, comorbidities, treatment effects and prognosis. Even though much knowledge regarding the IFN system in SLE has been accumulated during the last 16 years, much is still unclear or unknown. For instance, what is the cause or trigger of the IFN signature? To what extent contribute type II and type III IFN, besides type I IFN, to the IFN signature? Which cells produce the IFN, and are different cells responsible for the IFN production during different phases of the disease? Shall we block the IFN system in SLE, and if so, which is the most suitable target? We want to bring forward some aspects that are important, not at least for the understanding of how to stratify patients when deciding on line of therapy.

\section{Interferons}

IFNs constitute a fundamental part of the defence against viral infections and were originally defined by their ability to 'interfere' with viral replication. ${ }^{5}$ During viral infections, large amounts of IFNs are produced, activating the antiviral machinery in IFN-exposed cells, resulting in inhibition of viral replication. Viruses try to evade this innate defence mechanism and one noticeable example was the 'Spanish' influenza that could block expression of multiple IFN-stimulated genes, which probably contributed to the high mortality in the 1918 pandemic. ${ }^{6}$

There are three different types of IFNs (I-III), and the type I IFNs are the largest family. It can be divided into five classes (IFN- $\alpha, \beta, \varepsilon, \kappa$ and $\omega$ ), of which IFN- $\alpha$ can be further divided into 12 subtypes. $^{7}$ Most cells can produce small amounts of type I IFN, but the principal type I IFN-producing cell is the plasmacytoid dendritic cell (pDC), originally called the natural IFN-producing cell. ${ }^{8}$ The type I IFNs are typically induced by viruses, bacteria or microbial nucleic acids when sensed by pattern recognition receptors (PRRs) localised in the cytosol or in the endosome. These PRRS include Toll-like receptors 
Type III IFN

IFN入 1-4

Type I IFN

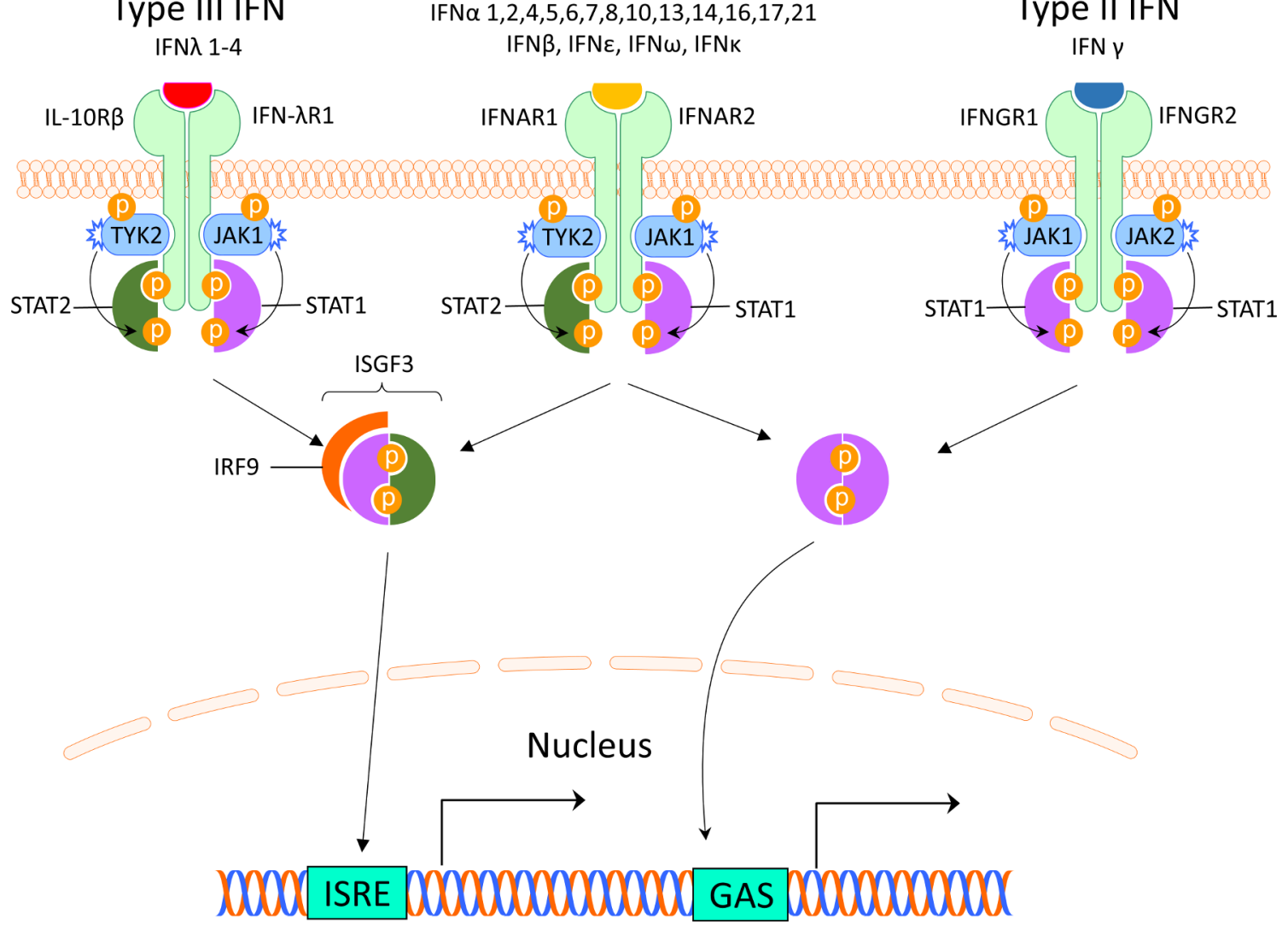

Figure 1 Interferon receptors and signalling. The interferons are classified into three types, which bind to distinct receptors. This induces activation of overlapping pathways resulting in expression of different genes. GAS, interferon-gamma activated sequence; IFN, interferon; IFNAR, interferon alpha receptor; IFNGR, interferon gamma receptor; IFN- $\lambda$ R1, interferon lambda receptor 1; IL-10R $\beta$, interleukin-10 receptor $\beta$; IRF, interferon regulatory factor; ISGF3, interferon-stimulated gene factor 3; ISRE, interferon-stimulated response elements; JAK, Janus kinase; STAT, signal transducer and activator of transcription; TYK2, tyrosine kinase 2.

(TLRs), retinoic acid inducible gene 1 (RIG-I)-like receptors (RLRs) and nucleotide oligomerisation domain-like receptors (NLRs). ${ }^{10}$ Type I IFNs all bind to the same ubiquitously expressed type I IFN receptor (IFNAR) that consists of two polypeptide chains of IFNAR1 and IFNAR2. The subsequent signalling pathway involves activation of Janus kinase (JAK) 1 and tyrosine kinase (TYK) 2 and formation of the interferon-stimulated gene factor 3-complex (IGSF3), including signal transducer and activator of transcription (STAT) 1, STAT2 and interferon regulatory factor (IRF) 9. IGSF3 binds to interferon stimulated response elements in promoters of IFN-regulated genes $^{11}$ (figure 1 ).

The type II IFN consists of only one member and many different cells produce IFN- $\gamma$ including natural killer (NK) cells and T cells. ${ }^{12}$ IFN- $\gamma$ binds to the IFN- $\gamma$ receptor (IFNGR) which is expressed on most cells. Via activation of JAK1 and JAK2, ligation of the IFNGR results in phosphorylation of STAT1 homodimers and binding to IFN- $\gamma$-activated sites (GASs) and subsequent gene expres$\operatorname{sion}^{11}$ (figure 1). This signalling pathway can also be used by IFNAR and there is therefore a large overlap between type I and II induced genes. ${ }^{13}$
Type III IFNs comprise four newly identified lambda IFNs: IFN $\lambda 1 /$ IL29, IFN $\lambda 2 /$ IL28A, IFN $\lambda 3 /$ IL28B and IFN $\lambda 4$ (IFNL4). ${ }^{14}$ IFN- $\lambda$ s are most abundant at barrier surfaces including the respiratory and gastrointestinal tracts, and is produced by epithelial and epithelial-origin cells including hepatocytes and some immune cells (macrophages and DCs). The type III IFNs signal through a receptor complex (IFNLR1/IL10R $\beta$ ) that is primarily expressed on epithelial cells (gastrointestinal, respiratory and urogenital), hepatocytes and a few immune cells including neutrophils and DCs ${ }^{14}$ (figure 1).

\section{Interferon in SLE}

Increased levels of IFN in serum of patients with SLE was already described 40 years ago ${ }^{15}$ and were later identified as type I IFNs. ${ }^{16}$ Observational studies found that patients treated for malignancies with IFN- $\alpha$ could develop a lupus-like disease with autoantibodies to nuclear antigens, suggesting that type I IFN can break the tolerance and induce an autoimmune disease. ${ }^{17} 18$ When genomewide expression analysis became available, several groups showed that $50 \%-75 \%$ of adult patients and up to $90 \%$ of children with SLE display an increased expression of 
type I IFN-regulated genes (an IFN signature).${ }^{1-4}$ Younger patients have a more prominent IFN activity compared with older patients, ${ }^{19}$ and SLE disease activity correlates with IFN- $\alpha$ levels and the strength of the IFN signature. $^{220-22}$ Analysis of longitudinal gene expression signatures suggest IFN- $\alpha$ to generate a relatively stable pattern of IFN-I transcripts over time, while other gene clusters may reflect induction of IFN- $\beta$ or IFN- $\gamma$ and present a more variable pattern. ${ }^{23}$ Thus, the IFN signature observed in patients with SLE probably corresponds to more IFN types than just IFN- $\alpha$, although the type I IFN seems most important.

It is important to notice that a very large number of genes are regulated by IFNs and the specific genes expressed depend on the cell type, expressed receptors, type of stimuli and timing of sampling. Recent studies have suggested that perhaps $10 \%$ of our genes are regulated by IFN, but type III IFN induces a limited number of genes and no unique transcripts have been defined. ${ }^{24}$ Studies of the IFN signature in SLE have included different compositions of cells, often whole blood or peripheral blood leucocytes, and the number of genes examined have varied from only a few to large panels. There is also a significant overlap between the genes induced by type I, II and III IFN, which is why it has been difficult to differentiate among the IFNs contributing to the signature. The results have been inconsistent and sometimes challenging to interpret, as no consensus on how to measure the score exists today. However, all three IFNs seem to contribute to the signature. ${ }^{232526}$ Perhaps not surprising, when comparing the IFN signature in patients with a viral infection and SLE, patients with SLE display a more complex pattern of gene expression. ${ }^{27}$ Studies of IFN expression in tissues have added further complexity to this field, as previous largely neglected IFNs may be important in specific organs, such as IFN- $\kappa$ in cutaneous lupus. $^{28}$

\section{Triggers of interferon production in SLE}

During the last years, a number of possible mechanisms explaining the persistent type I IFN production in SLE have been described, and in figure 2 several conceivable inducers of the IFN production are shown. An important mechanism of IFN- $\alpha$ induction is mediated via interferogenic ICs, which consist of autoantibodies and nucleic acid binding proteins. ${ }^{29-31}$ The ICs are endocytosed via

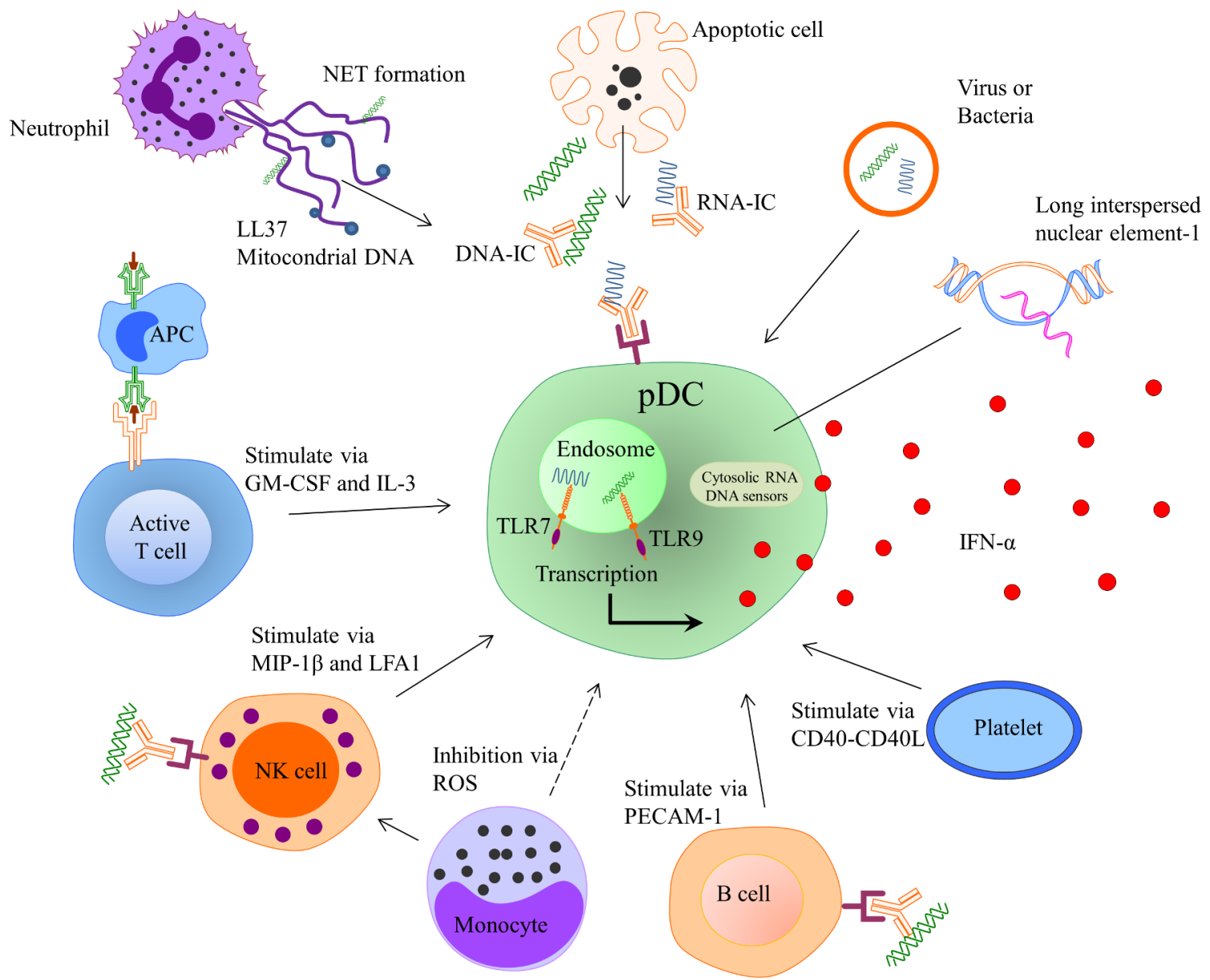

Figure 2 Inducers and regulators of IFN- $\alpha$ production by plasmacytoid dendritic cell. APC, antigen-presenting cell; GM-CSF, granulocyte-macrophage colony-stimulating factor; IC, immune complex; IFN, interferon; IL-3, interleukin 3; LFA1, Iymphocyte function-associated antigen 1; MIP-1 $\beta$, macrophage inflammatory protein-1 $\beta$; NET, neutrophil extracellular traps; PECAM-1, platelet and endothelial cell adhesion molecule 1; ROS, reactive oxygen species. 


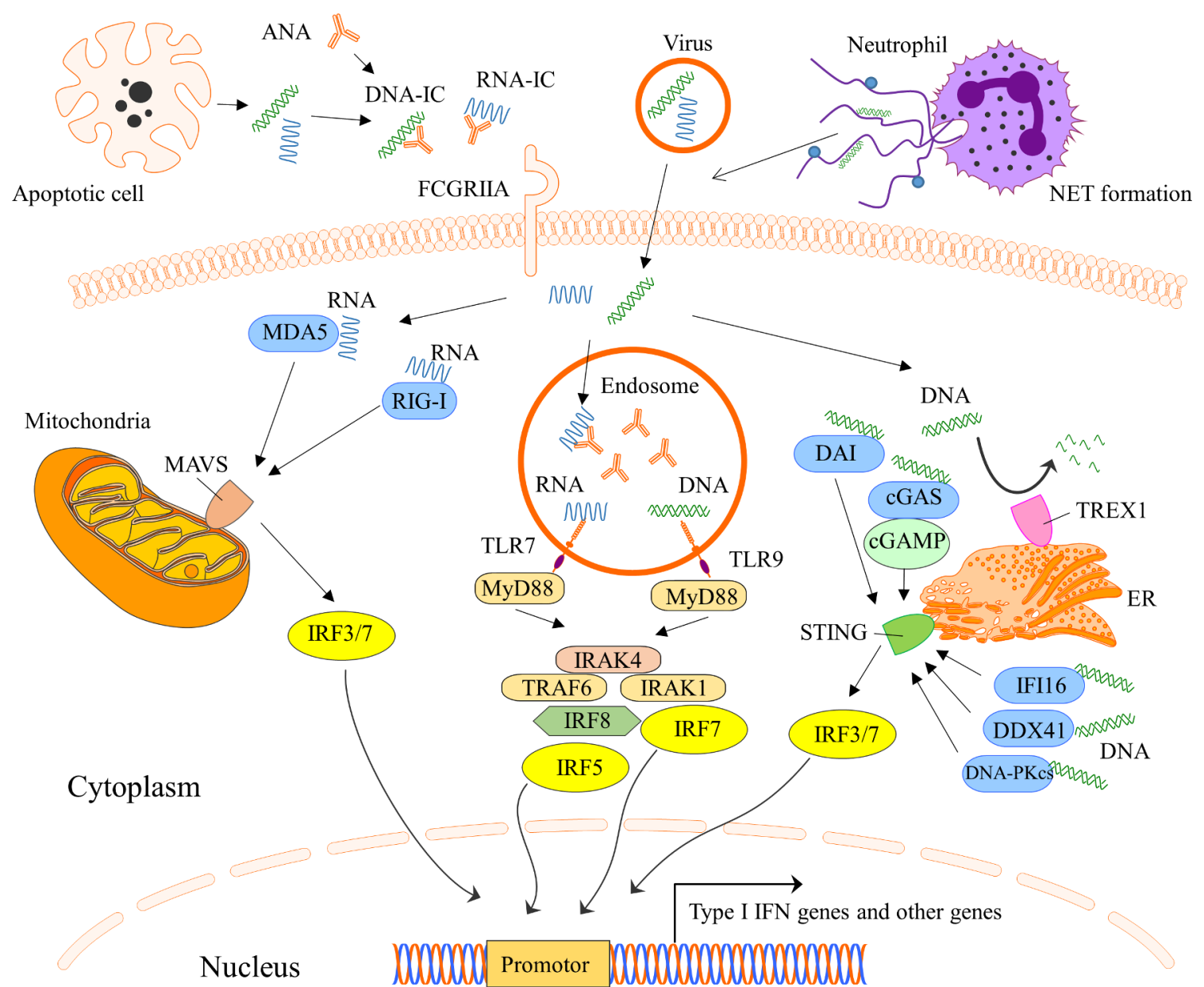

Figure 3 Schematic picture of the type I interferon production and different nucleic acid sensors. cGAMP, cyclic GMP-AMP; cGAS, cyclic GMP-AMP synthase; DAI, DNA-dependent activator of IFN-regulatory factors; DDX41, DEAD-box helicase 41; DNA-PKcs, DNA-dependent protein kinase; ER, endoplasmatic reticulum; FCGRIIA, Fc-gamma receptor II A; IC, immune complex; IFI16, gamma-interferon-inducible protein 16; IRAK, interleukin-1 receptor-associated kinase; IRF, interferon regulatory factor; MAVS, mitochondrial antiviral-signalling protein; MDA5, melanoma differentiation-associated protein 5; MyD88, myeloid differentiation primary response 88; NET, neutrophil extracellular traps; RIG-I, retinoic acid-inducible gene I; STING, stimulator of interferon genes; TLR, Toll-like receptor; TRAF6, TNF receptor-associated factors; TREX1, three prime repair exonuclease 1.

Fc $\gamma$ RIIa on pDCs, transported to the endosome where the nucleic acid part of the IC binds TLR7 or TLR9 with subsequent activation of transcription factors and IFN- $\alpha$ production $^{32}$ (figure 3 ). This route of IFN induction has been demonstrated in vitro, combining purified SLE IgG and apoptotic or necrotic cell material as well as small nuclear ribonucleoproteins (snRNPs), which is relevant given the increased apoptosis and reduced clearance of apoptotic debris observed in patients with SLE. ${ }^{33} 34$

NET formation is a cell death pathway where neutrophils extrude nuclear material such as histones, decondensed chromatin and cytoplasmatic proteins in a web-like structure. Patients with SLE have increased NET formation and an impaired capacity to degrade NETs due to decreased function of extracellular DNAse I. ${ }^{35}{ }^{36}$ This increases exposure of nucleic acids and proteins to autoreactive $\mathrm{B}$ cells and autoantibodies, and it has been shown that NETs activate pDCs to produce high levels of IFN- $\alpha$ in a TLR9-dependent manner ${ }^{36} 37$ (figure 2). Recently, it was shown that SLE neutrophils extrude high levels of oxidised mitochondrial DNA that can induce IFN- $\alpha$ production via the cGAS stimulator of interferon genes (STING) pathway. ${ }^{38}$ Furthermore, NET-derived cationic antimicrobial peptide LL37-DNA complexes expand self-reactive memory B cells to produce anti-LL37 Abs in an Ag-dependent manner. ${ }^{39}$ Highlighting the importance of exposure of autoantigens to the immune system for IC formation and IFN induction, other described self-derived IFN inducers include high mobility group box chromosomal protein and heat shock protein $90 .{ }^{40-42}$

Transposable elements (TA) are DNA sequences that can change position within a genome and constitutes more than half of the human DNA. ${ }^{43}$ It represents a potential significant source of stimulatory self-nucleic acid. Long interspersed nuclear element-1 (L1) is a class I TA and 80-100 L1s are thought to remain active in any given individual. ${ }^{43}$ Hypomethylation of L1 has been observed in SLE and is associated with increased L1 expression. ${ }^{445} \mathrm{In}$ 
kidney biopsies of patients with SLE nephritis, increased expression of $\mathrm{L} 1$ has been observed and correlates with expression of type I IFN. ${ }^{45}$ L1 triggers IFN production by pDCs via TLR7 but also by monocytes via cytoplasmic RIG-I. ${ }^{45}$

Infections can trigger the onset of SLE or a disease flare, and although many viruses have been implicated in the aetiology of SLE, no specific virus or bacteria has been identified to cause the disease. Gut microbiota can activate STING and induce type I IFN, ${ }^{46}$ and recently it was shown that the Gram-positive bacteria Enterococcus gallinatum translocate across the gut barrier and induce Th17 and Tfh cells as well as innate immune pathways including the pDC/IFN axis. ${ }^{47}$ Treatment of the lupusprone mice $(\mathrm{NZW} \times \mathrm{BXSB}) \mathrm{F} 1$ with antibiotics inhibited autoantibody production and ameliorated disease. Given the impaired gut barrier function observed in SLE, these data highlight the possibility that the gut microbiome could be of aetiopathogenetic importance in at least a subset of patients with SLE.

In conclusion, there exist a large number of possible inducers of IFN production in SLE and probably different inducers are most important in different patients. Greater understanding of the relevant trigger(s) and pathways mediating the IFN production in individual patients would be of great help in order to develop precise treatments that target the specific IFN inducers causing a persistent IFN production.

\section{Interferon-producing cells in SLE}

The number of pDCs is reduced in the circulation of patients with SLE, but can be detected in inflamed tissues, such as $\operatorname{skin}^{48} 49$ and kidneys, where they seem to be activated. ${ }^{50}$ Several lines of evidence suggest that $\mathrm{pDCs}$ to a great extent are responsible for the ongoing IFN production in SLE. Thus, in murine models of lupus, depletion of pDC ameliorates the disease ${ }^{51}$ and genetically impaired $\mathrm{pDC}$ function improves the disease. ${ }^{52} \mathrm{~A}$ recent study also showed that targeting pDC in patients with SLE decreases the expression of IFN response genes in blood, reduces cutaneous immune cell infiltrates and ameliorates skin lesions. ${ }^{53}$ Despite these observations, the role of other IFN-producing cells in SLE needs to be clarified because a number of studies suggest that several other cell types are involved in IFN production. Among these are the keratinocytes, which can produce both IFN- $\kappa^{28}$ and IFN- $\lambda^{26}$ and monocytes, which have been implicated in the generation of the IFN signature. ${ }^{54}$ Monocytes are mainly responsible for IFN production in the pristaine-induced murine lupus model, which is characterised by a prominent IFN signature, and human monocyte-derived macrophages transfected with a small non-coding Y RNA or stimulated with immune complexes express IFN- $\alpha$ and IFN- $\beta$ mRNA. ${ }^{55}$ However, the precise role of IFN-producing monocytes in human SLE is at the moment unresolved and needs to be further explored. Neutrophils have the capacity to produce type I IFN ${ }^{56}$ and bone marrow-derived neutrophils in patients with SLE produce IFN- $\alpha .{ }^{57}$ Produced IFN seems to promote alterations in B-cell development with a reduction in the fraction of pro/pre-B cells, suggesting an inhibition in early B-cell development and an expansion of B cells at the transitional stage. This could well be an early event in the breakage of tolerance and development of autoimmunity with autoantibody production. Besides type I IFN-producing cells, activated NK cells in SLE have an increased production of IFN- $\gamma^{58}$ Furthermore, detectable IFN- $\lambda$ transcripts have been noted in peripheral blood leucocytes from patients with SLE, ${ }^{59}$ but the exact source of IFN- $\lambda$ in patients with SLE is at the moment unclear.

An important observation is that several cell types, once activated, can stimulate $\mathrm{pDC}$ to an increased IFN production. Thus, NK cells, B cells and T cells all can enhance IFN production when pDCs are exposed to nucleic acid-containing immune complexes ${ }^{60-62}$ (figure 2). The in vivo relevance of these findings remains to be established, but suggests that in SLE there is an extensive crosstalk between different immune cells and pDCs, which promotes the ongoing IFN production and sustained autoimmune process.

In summary, several cell types can contribute to the IFN signature seen in patients with SLE, and although pDC most probably is the main source of the IFN, it seems conceivable that in a subset of patients, other cell types are important IFN producers that need to be targeted in order to completely control the activated IFN system.

\section{Genetic factors influencing the IFN system in SLE}

Today, more than 100 genetic risk loci have been associated to $\mathrm{SLE}^{63}$ and more than half of the identified SLE susceptibility genes encode proteins with functions directly or indirectly linked to type I IFN production or responses. ${ }^{64} 65$ These include genes involved in Toll-like receptor activation and their downstream signalling molecules. For most of the risk gene variants, the mechanism by which the risk gene contributes to disease susceptibility, or severity, is unknown, but recent studies have shed some light on this issue. One of the strongest SLE risk loci outside the HLA region is signal transducer and activator of transcription (STAT) 4, which has been known as a SLE risk locus for more than 10 years. ${ }^{66}$ The intronic SLE-associated STAT4 SNPs are linked to a disease phenotype with an earlier onset and an increased risk for stroke and nephritis with severe renal insufficiency. ${ }^{67-69}$ We recently showed that activated $\mathrm{T}$ cells from STAT4 risk allele carriers with SLE have increased levels of STAT4 protein, resulting in more phosphorylated STAT4 in response to IL-1 2 and IFN- $\alpha$, and an augmented IL-12-induced IFN- $\gamma$ production. ${ }^{70}$ In contrast, activated $\mathrm{T}$ cells from healthy individuals carrying the STAT4 risk allele displayed a decreased response. This finding may be of importance as to why the majority of risk allele carriers do not develop disease and suggests that the STAT4 risk allele needs to interact with other host or environmental factors to be pathogenic. As pre-incubation of healthy donor cells with IFN- $\alpha$ selectively enhanced the IL-12 response in STAT4 
risk allele carriers, it is possible that STAT4 risk allele carriers are at risk to develop SLE during prolonged type I IFN production. ${ }^{71}$ These results also link a SLE susceptibility locus to both the type I and type II IFN systems, and one could speculate that these findings at least partly can explain the good therapeutic response to the anti-IL-12/ IL-23 mAb ustekinumab in a proportion of patients with SLE. ${ }^{72}$

The interferonopathies comprise a group of rare monogenic diseases with a constitutive overproduction of type I IFN caused by mutations in genes responsible for handling of nucleic acids. ${ }^{73}$ Thus, these patients have various disturbances in the intracellular nucleic acid metabolism or in cytosolic nucleic acid-sensing pathways, which cause an autoimmune or autoinflammatory disease. The patients have a prominent IFN signature, but show a remarkable phenotypic heterogeneity, which indicates that other genes and environmental factors modify the inflammatory response. Some of the patients have a clear SLE phenotype, and it is possible that genes responsible for the interferonopathies also contribute to the development of the disease in a subset of patients with SLE normally encountered at the rheumatology department. In fact, a recent study of whole-genome sequencing of patients with SLE shows that ultra-rare, coding heterozygous variant connected to the diverse spectrum of interferonopathies are over-represented among patients with SLE. ${ }^{74}$

Epigenetic changes are prominent in cells and tissues from patients with SLE and a recent, comprehensive review summarises DNA methylation studies in SLE. ${ }^{75}$ Patients with SLE have decreased methylation levels in a large number of CpG sites and the most strongly hypomethylated genes are IFN regulated. ${ }^{76}$ Furthermore, in a study of twins discordant for SLE, there was a higher degree of hypomethylation in IFN-regulated genes among twins that had experienced a flare within the past 2 years, which link the epigenetic IFN signature to a more active disease. ${ }^{77}$ Since some epigenetic changes are mitotically heritable and relatively stable, it is possible that some of the demethylated sites in IFN-regulated genes are hypomethylated already in utero, or received from one of the parents, although IFN exposure during disease flares seems most plausible.

Taken together, genetic studies demonstrate that the genetic risk for development of SLE is strongly connected to gene variants in the IFN signalling pathway and changes in IFN-regulated genes. The mechanisms by which these alterations are involved in the development of SLE are under intense studies, but results so far strengthen the assumption that the genetic setup directly contributes to an IFN-driven autoimmune process.

\section{Connection between the IFN system and other immune cells}

As mentioned above, a number of cells in the immune system can interact with $\mathrm{pDC}$ and enhance the IFN response. Perhaps even more important are the effects of produced IFN on most cells in both the innate and adaptive immune systems (reviewed in Eloranta $e t a l^{8}$ ). Type I IFN acts as an immune adjuvant and one mechanism for the enhanced immune response by type I IFN is an increased expression of MHC I molecules, ${ }^{78}$ which facilities the cross-presentation of exogenous antigens as well as detection of virus-infected cells by cytotoxic $\mathrm{T}$ cells. IFN also promotes the expression of a number of other molecules important in the immune response, such as MHC II, CD40, CD80 and CD86, but also the expression of chemokines and their cognate receptors such as CXCL10 and CXCR3. DCs stimulated by IFN mature and achieve a molecular repertoire that makes them very potent as antigen-presenting cells and capable to induce differentiation of naïve $\mathrm{CD} 4^{+} \mathrm{T}$ cells, but also development of $\mathrm{CD}^{+}$memory T cells. Type I IFN increases the differentiation of Th17 cells and suppress Treg functions, which can all lead to an expansion of autoreactive T cells ${ }^{79}$

Type I IFNs impact B-cell function through a variety of mechanisms that lead to prolonged survival and activation, including differentiation and class-switch recombination causing enhanced antibody production (reviewed in Kiefer $e t a l^{80}$ ). Type I IFNs increase the production of B-cell activating factor in monocytes and via this mechanism stimulate antibody production. ${ }^{81}$ NK cells are considered important in at least a subgroup of patients with $\mathrm{SLE}^{82}$ and type I IFNs increase the cytotoxicity and IFN- $\gamma$ production in NK cells, linking both the type I and type II IFN system to each other. ${ }^{83}$

The type I IFNs also have effects outside the immune system, such as impairment of endothelium-dependent vasorelaxation and endothelial progenitor cell function, which slow down the repair process of damaged endothelium. ${ }^{84-86}$ Further, IFN- $\alpha$ inhibits eNOS expression and impairs insulin-mediated nitric oxide production in endothelial cells, ${ }^{87}$ enhances foam cell formation ${ }^{88}$ and alters platelet function. ${ }^{89}$ These observations can be linked to the unexpectedly high prevalence of atherosclerosis and cardiovascular disease in patients with SLE. ${ }^{90}$ In a more general perspective, it is clear that there are several detrimental effects on cells and tissues that are exposed to IFN for an extensive period of time. ${ }^{91}$ Figure 4 summarises the effect of various IFNs on different cell types. ${ }^{14259293}$

\section{Disease process in SLE}

The many findings concerning the IFN system in patients with SLE can be put together into an aetiopathogenic model of SLE, which has been reviewed elsewhere ${ }^{94}$ For instance, an initial infection by a virus can induce type I IFN production and release of cellular material from dying cells. Several other triggers of IFN production also exist, as discussed above. The extracellular autoantigens from apoptotic and necrotic cells as well as NETs from granulocyte then trigger B cells to autoantibody production against RNA and DNA binding proteins in individuals prone to autoimmune reactions. ICs will be formed, which act as endogenous type I IFN inducers, causing a prolonged stimulation of type I IFN production by pDCs. The excessive release of endogenous DNA/RNA 


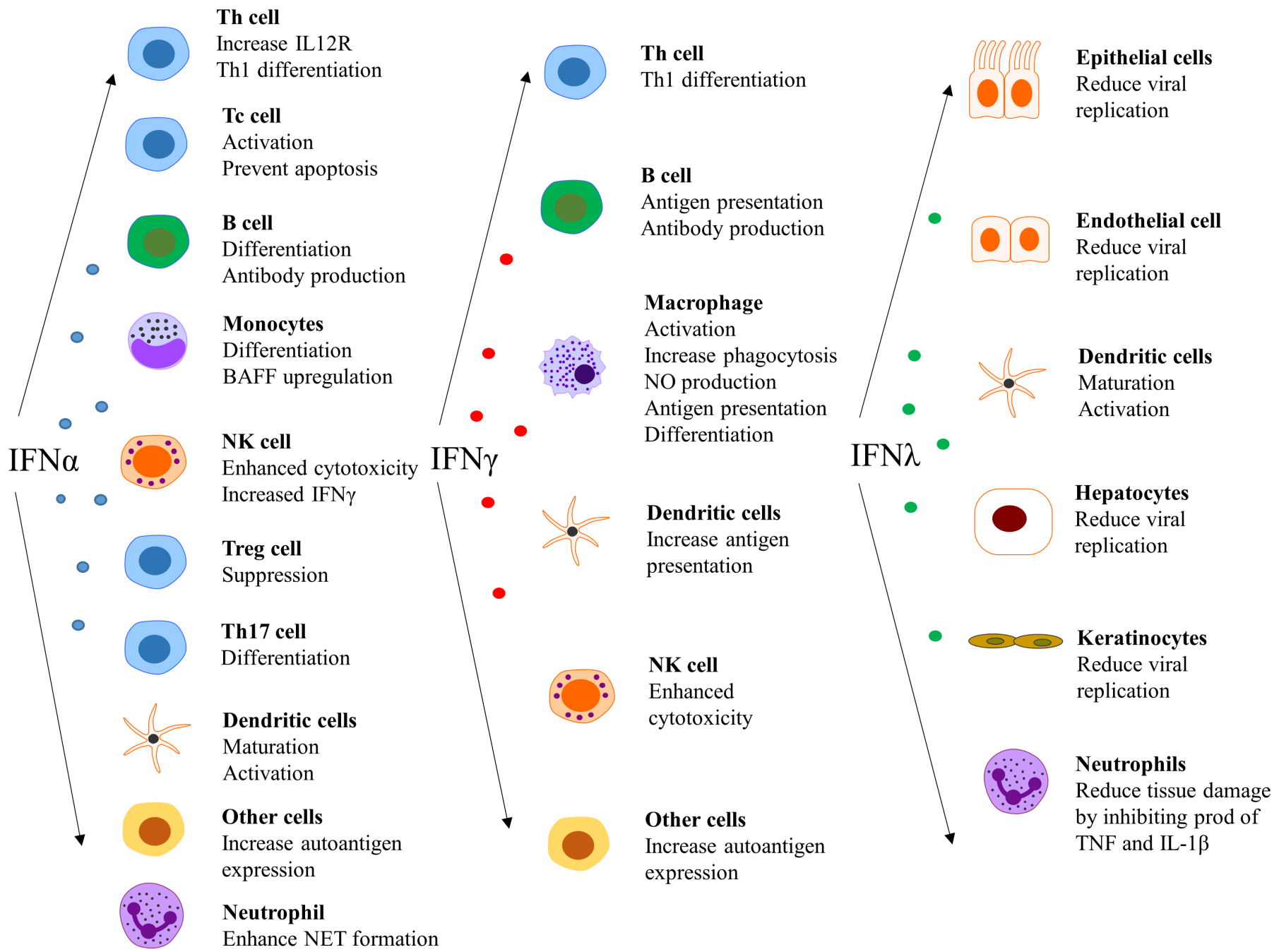

Figure 4 Effect of interferons (IFNs) on different cell types. NET, neutrophil extracellular traps.

in combination with impaired clearance of apoptotic cell material will facilitate the production of IFN by a number of cell types. This will result in chronic activation of the IFN system, which will drive an autoimmune process leading to chronic inflammation and tissue damage in a vicious circle manner.

\section{IFN system and disease manifestations}

A number of signs and symptoms in patients with SLE are connected to the increased production of IFN. General symptoms of acute viral infections such as muscle and joint pain, headache, pleurisy, fatigue and fever are associated with type I IFN. ${ }^{20}$ IFN also has a suppressive effect on the bone marrow resulting in anaemia, neutropenia, lymphopenia and thrombocytopenia, ${ }^{95}$ and consequently a high IFN signature score has been associated with the haematological American College of Rheumatology criteria. ${ }^{2}$ Presence of anti-dsDNA, anti-SSA and anti-RNP is associated with high IFN- $\alpha$ activity ${ }^{96}$ and antibodies to RNA with a strong IFN signature, ${ }^{21}$ highlighting the link between IFN and B-cell maturation and autoantibody production.
Skin

Patients with hereditary interferonopathies often present with cutaneous manifestations including malar rash and alopecia. ${ }^{97}$ In SLE, the IFN signature correlates with cutaneous disease activity ${ }^{98}$ and IFN-regulated genes are expressed in epidermis and dermis of cutaneous lesions. ${ }^{28} 99$ Increased expression of IFN- $\lambda 1^{26}$ and IFNK ${ }^{28}$ has been observed in the skin of patients with cutaneous SLE and type I IFN has been suggested to inhibit ADAM17 in Langerhans cells thereby enhancing UVB-induced keratinocyte apoptosis. ${ }^{100}{ }^{101}$ Recent phase I-II trials in SLE blocking IFN signalling via $\mathrm{pDC},{ }^{53}$ IFNAR $^{102}$ or the JAK/STAT pathway ${ }^{103}$ have all improved skin manifestations and JAK inhibition has also improved skin manifestations in patients with interferonopathies. ${ }^{104}$ Thus, IFN signalling seems to be a key player in SLE skin pathology even though the exact interplay between different IFNs, keratinocytes and pDCs needs further exploration.

Arthritis

Synovial tissue from patients with lupus with inflammatory arthritis demonstrate increased expression of IFN-induced genes, ${ }^{105}$ and recent data suggest IFN signatures 
induced by IFN- $\beta 1$ and IFN- $\alpha 2$ to be most important. ${ }^{106}$ The source of IFN in the synovium is not clear, but fibroblast-producing IFN- $\beta$, which are rich in this tissue, have been suggested. ${ }^{106}{ }^{107}$ Results from the Anifrolumab trial, blocking IFNAR, show significant improvement of joint disease in patients with lupus with an initially strong IFN signature, indicating an important role for IFNs in lupus arthritis. ${ }^{102}$

\section{Kidneys}

Regarding SLE nephritis, studies have shown an association between a strong IFN signature and both a history of nephritis ${ }^{21}$ and active nephritis. ${ }^{22}$ Kidney biopsies of patients with SLE show increased expression of IFN-inducible genes ${ }^{108-110}$ and pDCs accumulate in glomeruli of patients with active disease.$^{50} \mathrm{~A}$ stronger IFN signature is observed in glomeruli and renal tubule in patients with immunosuppressive naive SLE with nephritis compared with patients with SLE nephritis having received immunosuppressive treatment. ${ }^{111}$ In kidney tissue, IFN- $\beta$ can induce podocyte cell death and increase permeability whereas both IFN- $\alpha$ and IFN- $\beta$ suppress renal progenitor cell differentiation into mature podocytes, ${ }^{112}$ resulting in podocyte loss, proteinuria and impaired glomerular repair. Taken together, these observations suggest that IFN is important in both the inflammatory process and development of damage in SLE nephritis.

\section{Central nervous system}

Increased levels of type I IFN have been demonstrated in the cerebrospinal fluid of patients with SLE with neuropsychiatric manifestations, ${ }^{113}$ including lupus psychosis ${ }^{114}$ and also in the central nervous system (CNS) post mortem. ${ }^{114}$ This is intriguing given the observed adverse neuropsychiatric effects following IFN- $\alpha$ treatment. ${ }^{115}$ Locally produced cerebrospinal fluid autoantibodies from patients with CNS lupus can form ICs and stimulate IFN- $\alpha$ production by $\mathrm{pDCs}^{116}$ and type I IFN stimulates microglia to become reactive and engulf neuronal and synaptic material in lupus-prone mice. ${ }^{117}$ Thus, it is possible that interferogenic ICs and type I IFN can be of importance for the neuropsychiatric (NP) manifestations often observed in SLE and NP-SLE may be one manifestation of the disease suitable for IFN inhibition.

\section{Targeting the IFN system}

After the discovery of the IFN signature, a number of different strategies have been developed in order to downregulate the IFN system in patients with SLE. So far, the therapeutic effect has been modest and difficult to reproduce in larger phase III studies. ${ }^{118}$ There are several reasons for this, but there are a number of factors that need to be taken into consideration before selecting therapeutic target in a patient with SLE. Several have been discussed above and some are summarised in table 1 .

Recent clinical trials have stratified patients by clinical manifestations, including nephritis or skin and joint manifestations. Unfortunately, several trials have failed, which is why in the selection of patients, the molecular pathways activated in a single patient must also be taken into consideration. In this context, it is important to note that the type I IFN system may be most critical early in the disease process ${ }^{218119120}$ and at initiation of flares. ${ }^{20}$ Later in the disease course, other IFN subtypes may have a more prominent role, at least in some patients. ${ }^{72}$ Therefore, defining the IFN and pathway activation of importance for individual patients will be necessary. This analysis also includes the many pathways related to the IFN system. Attempts have been made to refine the IFN signature using factor analysis and by linking ISG expression to IFN subtype. ${ }^{23} 121$ Others have applied single-cell RNA sequencing of lupus kidney biopsies, identifying a high IFN response signature in tubular cells as a negative prognostic marker of lupus nephritis. ${ }^{122}$ Further studies of how specific cells and cell types contribute to the disease process and organ manifestations may reveal how to personalise the treatment for the patients and also on the cellular level.

Genetic profiling will also help to determine the underlying mechanism of disease in single patients. Individuals with rare monogenic SLE, including patients with rare variants of genes linked to interferonopathies, ${ }^{74}$ or genetic complement deficiency may benefit from individualised treatment. As discussed above, patients with risk gene variants linked to IFN- $\gamma$ signalling including STAT4 and IL12 might benefit from inhibition of this pathway. In the future, it will perhaps be important to consider the cumulative genetic risk, as defined by a genetic risk score, when selecting therapy as this may predict disease outcome. ${ }^{123}$ In the years to come, we expect that genetic studies (genotype-phenotype) will give us more

Table 1 Factors to consider before selecting the therapeutic target in a patient with SLE

$\begin{array}{ll}\text { Timing } & \text { Type I IFN activation is most prominent early in the disease process and during some flares } \\ \text { IFN inducer } & \text { Several IFN inducers exist and may differ between patients. Prevent generation of } \\ & \text { IFN inducers or degrade triggers? Block sensors of IFN inducers? } \\ \text { IFN-producing cells } & \text { Inhibit pDCs and/or other cell types as well? } \\ \text { Type of IFN } & \text { Which IFN(s) are most relevant in a specific patient? } \\ \text { IFN target } & \text { Block IFN receptor(s) or signalling pathways? }\end{array}$

IFN, interferon; pDC, plasmacytoid dendritic cell. 
information about what pathways to target in the individual patient.

\section{CONCLUSION}

The IFN system is our most fundamental defence system against infections, but in patients with SLE, there is an ongoing production of IFN that sustains an autoimmune process. The complexity of the IFN system, together with the many clinical features of SLE, has made it difficult to target the proper molecules in single patients. However, during the last years, we have seen a dramatic increase in the understanding of the IFN system and its role in SLE. Although this information has added more elements to consider in our clinical decision process, we are now closer than ever to unlock the mystery of how to target the IFN pathway in SLE.

Acknowledgements We would like to acknowledge the critical review of the manuscript by Niklas Hagberg and Maija-Leena Eloranta.

Contributors LR and DL wrote and approved the manuscript.

Funding The study was supported by the Swedish Rheumatism Association, King Gustaf V's 80-years Foundation, the Swedish Research Council and the Swedish Society of Medicine (the Ingegerd Johansson donation).

Competing interests $L R$ has received a research grant from AstraZeneca and received honoraria for scientific advice from Biogen.

Patient consent for publication Not required.

Provenance and peer review Commissioned; externally peer reviewed. Data availability statement No additional data are available.

Open access This is an open access article distributed in accordance with the Creative Commons Attribution Non Commercial (CC BY-NC 4.0) license, which permits others to distribute, remix, adapt, build upon this work non-commercially, and license their derivative works on different terms, provided the original work is properly cited, appropriate credit is given, any changes made indicated, and the use is non-commercial. See: http://creativecommons.org/licenses/by-nc/4.0/.

\section{REFERENCES}

1. Bennett L, Palucka AK, Arce E, et al. Interferon and granulopoiesis signatures in systemic lupus erythematosus blood. J Exp Med 2003;197:711-23.

2. Baechler EC, Batliwalla FM, Karypis G, et al. Interferon-inducible gene expression signature in peripheral blood cells of patients with severe lupus. Proc Natl Acad Sci U S A 2003;100:2610-5.

3. Crow MK, Kirou KA, Wohlgemuth J. Microarray analysis of interferon-regulated genes in SLE. Autoimmunity 2003;36:481-90.

4. Han G-M, Chen S-L, Shen N, et al. Analysis of gene expression profiles in human systemic lupus erythematosus using oligonucleotide microarray. Genes Immun 2003;4:177-86.

5. Isaacs $A$, Lindenmann J. Virus interference. I. The interferon. Proc $R$ Soc Lond B Biol Sci 1957;147:258-67.

6. Billharz R, Zeng H, Proll SC, et al. The NS1 protein of the 1918 pandemic influenza virus blocks host interferon and lipid metabolism pathways. J Virol 2009;83:10557-70.

7. Pestka S, Krause CD, Walter MR. Interferons, interferon-like cytokines, and their receptors. Immunol Rev 2004;202:8-32.

8. Eloranta M-L, Alm GV, Rönnblom L. Disease mechanisms in rheumatology - tools and pathways: plasmacytoid dendritic cells and their role in autoimmune rheumatic diseases. Arthritis Rheum 2013;65:853-63.

9. Rönnblom L, Alm GV. A pivotal role for the natural interferon $\alpha-$ producing cells (plasmacytoid dendritic cells) in the pathogenesis of lupus. J Exp Med 2001;194:F59-64.

10. Barrat FJ, Elkon KB, Fitzgerald KA. Importance of nucleic acid recognition in inflammation and autoimmunity. Annu Rev Med 2016;67:323-36.

11. Platanias LC. Mechanisms of type-I- and type-Il-interferonmediated signalling. Nat Rev Immunol 2005;5:375-86.
12. Lee AJ, Ashkar AA. The dual nature of type I and type II interferons. Front Immunol 2061;2018.

13. Hertzog P, Forster S, Samarajiwa S. Systems biology of interferon responses. J Interferon Cytokine Res 2011;31:5-11.

14. Andreakos E, Zanoni I, Galani IE. Lambda interferons come to light: dual function cytokines mediating antiviral immunity and damage control. Curr Opin Immunol 2019;56:67-75.

15. Hooks JJ, Moutsopoulos HM, Geis SA, et al. Immune interferon in the circulation of patients with autoimmune disease. N Engl J Med 1979;301:5-8.

16. Ytterberg SR, Schnitzer TJ. Serum interferon levels in patients with systemic lupus erythematosus. Arthritis Rheum 1982;25:401-6.

17. Rönnblom LE, Alm GV, Oberg KE. Autoimmunity after alphainterferon therapy for malignant carcinoid tumors. Ann Intern Med 1991;115:178-83.

18. Rönnblom LE, Alm GV, Oberg KE. Possible induction of systemic lupus erythematosus by interferon- $\alpha$ treatment in a patient with a malignant carcinoid tumour. J Intern Med 1990;227:207-10.

19. Niewold TB, Adler JE, Glenn SB, et al. Age- and sex-related patterns of serum interferon- $\alpha$ activity in lupus families. Arthritis Rheum 2008;58:2113-9.

20. Bengtsson AA, Sturfelt $G$, Truedsson L, et al. Activation of type I interferon system in systemic lupus erythematosus correlates with disease activity but not with antiretroviral antibodies. Lupus 2000;9:664-71.

21. Kirou KA, Lee C, George S, et al. Activation of the interferon- $\alpha$ pathway identifies a subgroup of systemic lupus erythematosus patients with distinct serologic features and active disease. Arthritis Rheum 2005;52:1491-503.

22. Feng X, Wu H, Grossman JM, et al. Association of increased interferon-inducible gene expression with disease activity and lupus nephritis in patients with systemic lupus erythematosus. Arthritis Rheum 2006;54:2951-62.

23. Chiche L, Jourde-Chiche N, Whalen E, et al. Modular transcriptional repertoire analyses of adults with systemic lupus erythematosus reveal distinct type I and type II interferon signatures. Arthritis Rheumatol 2014;66:1583-95.

24. Schoggins JW. Interferon-stimulated genes: what do they all do? Annu Rev Virol 2019;6.

25. Rönnblom L, Eloranta $M-L$. The interferon signature in autoimmune diseases. Curr Opin Rheumatol 2013;25:248-53.

26. Zahn S, Rehkämper C, Kümmerer BM, et al. Evidence for a pathophysiological role of keratinocyte-derived type III interferon (IFN $\lambda$ ) in cutaneous lupus erythematosus. J Invest Dermatol 2011;131:133-40.

27. Kyogoku C, Smiljanovic B, Grün JR, et al. Cell-specific type I IFN signatures in autoimmunity and viral infection: what makes the difference? PLoS One 2013;8:e83776.

28. Sarkar MK, Hile GA, Tsoi LC, et al. Photosensitivity and type I IFN responses in cutaneous lupus are driven by epidermal-derived interferon kappa. Ann Rheum Dis 2018;77:1653-64.

29. Cederblad B, Blomberg S, Vallin $\mathrm{H}$, et al. Patients with systemic lupus erythematosus have reduced numbers of circulating natural interferon- $\alpha$ - producing cells. J Autoimmun 1998;11:465-70.

30. Vallin H, Blomberg S, Alm GV, et al. Patients with systemic lupus erythematosus (SLE) have a circulating inducer of interferon-alpha (IFN-alpha) production acting on leucocytes resembling immature dendritic cells. Clin Exp Immunol 1999;115:196-202.

31. Vallin H, Perers A, Alm GV, et al. Anti-double-stranded DNA antibodies and immunostimulatory plasmid DNA in combination mimic the endogenous IFN-alpha inducer in systemic lupus erythematosus. J Immunol 1999;163:6306-13.

32. Båve $U$, Magnusson $M$, Eloranta $M-L$, et al. Fc $\gamma R$ Ila is expressed on natural IFN- $\alpha$-producing cells (plasmacytoid dendritic cells) and is required for the IFN- $\alpha$ production induced by apoptotic cells combined with lupus IgG. J Immunol 2003;171:3296-302.

33. Båve U, Alm GV, Rönnblom L. The combination of apoptotic U937 cells and lupus IgG is a potent IFN- $\alpha$ inducer. $J$ Immunol 2000;165:3519-26.

34. Lövgren T, Eloranta M-L, Båve $U$, et al. Induction of interferon- $\alpha$ production in plasmacytoid dendritic cells by immune complexes containing nucleic acid released by necrotic or late apoptotic cells and lupus IgG. Arthritis Rheum 2004;50:1861-72.

35. Leffler J, Ciacma K, Gullstrand B, et al. A subset of patients with systemic lupus erythematosus fails to degrade DNA from multiple clinically relevant sources. Arthritis Res Ther 2015;17.

36. Lande R, Ganguly D, Facchinetti V, et al. Neutrophils activate plasmacytoid dendritic cells by releasing self-DNA-peptide complexes in systemic lupus erythematosus. Sci Transl Med 2011;3:73ra19. 
37. Garcia-Romo GS, Caielli S, Vega B, et al. Netting neutrophils are major inducers of type I IFN production in pediatric systemic lupus erythematosus. Sci Transl Med 2011;3:73ra20.

38. Lood C, Blanco LP, Purmalek MM, et al. Neutrophil extracellular traps enriched in oxidized mitochondrial DNA are interferogenic and contribute to lupus-like disease. Nat Med 2016;22:146-53.

39. Gestermann N, Di Domizio J, Lande R, et al. Netting neutrophils activate autoreactive B cells in lupus. J Immunol 2018;200:3364-71.

40. Tian J, Avalos AM, Mao S-Y, et al. Toll-like receptor 9-dependent activation by DNA-containing immune complexes is mediated by HMGB1 and RAGE. Nat Immunol 2007;8:487-96.

41. Okuya K, Tamura Y, Saito K, et al. Spatiotemporal regulation of heat shock protein 90-chaperoned self-DNA and CpGoligodeoxynucleotide for type I IFN induction via targeting to static early endosome. J Immunol 2010;184:7092-9.

42. Lande R, Gregorio J, Facchinetti V, et al. Plasmacytoid dendritic cells sense self-DNA coupled with antimicrobial peptide. Nature 2007;449:564-9.

43. Hancks DC, Kazazian HH. Roles for retrotransposon insertions in human disease. Mob DNA 2016;7:9.

44. Nakkuntod J, Avihingsanon Y, Mutirangura A, et al. Hypomethylation of LINE-1 but not Alu in lymphocyte subsets of systemic lupus erythematosus patients. Clinica Chimica Acta 2011:412:1457-61.

45. Mavragani CP, Sagalovskiy I, Guo Q, et al. Expression of long interspersed nuclear element 1 retroelements and induction of type I interferon in patients with systemic autoimmune disease. Arthritis Rheumatol 2016;68:2686-96.

46. Canesso MCC, Lemos L, Neves TC, et al. The cytosolic sensor sting is required for intestinal homeostasis and control of inflammation. Mucosal Immunol 2018;11:820-34.

47. Manfredo Vieira S, Hiltensperger M, Kumar V, et al. Translocation of a gut pathobiont drives autoimmunity in mice and humans. Science 2018;359:1156-61.

48. Blomberg S, Eloranta ML, Cederblad B, et al. Presence of cutaneous interferon-alpha producing cells in patients with systemic lupus erythematosus. Lupus 2001;10:484-90.

49. Farkas L, Beiske K, Lund-Johansen F, et al. Plasmacytoid dendritic cells (natural interferon- $\alpha / \beta$-producing cells) accumulate in cutaneous lupus erythematosus lesions. Am J Pathol 2001;159:237-43.

50. Tucci M, Quatraro C, Lombardi L, et al. Glomerular accumulation of plasmacytoid dendritic cells in active lupus nephritis: role of interleukin-18. Arthritis Rheum 2008;58:251-62.

51. Rowland SL, Riggs JM, Gilfillan S, et al. Early, transient depletion of plasmacytoid dendritic cells ameliorates autoimmunity in a lupus model. J Exp Med 2014;211:1977-91.

52. Sisirak V, Ganguly D, Lewis KL, et al. Genetic evidence for the role of plasmacytoid dendritic cells in systemic lupus erythematosus. $J$ Exp Med 2014;211:1969-76.

53. Furie R, Werth VP, Merola JF, et al. Monoclonal antibody targeting BDCA2 ameliorates skin lesions in systemic lupus erythematosus. $J$ Clin Invest 2019;129:1359-71.

54. Porat A, Giat E, Kowal C, et al. DNA-mediated interferon signature induction by SLE serum occurs in monocytes through two pathways: a mechanism to inhibit both pathways. Front Immunol 2018;9:2824.

55. Clancy RM, Markham AJ, Reed JH, et al. Targeting downstream transcription factors and epigenetic modifications following Toll-like receptor $7 / 8$ ligation to forestall tissue injury in anti-Ro60 associated heart block. J Autoimmun 2016;67:36-45.

56. Denny MF, Yalavarthi S, Zhao W, et al. A distinct subset of proinflammatory neutrophils isolated from patients with systemic lupus erythematosus induces vascular damage and synthesizes type I IFNs. J Immunol 2010;184:3284-97.

57. Palanichamy A, Bauer JW, Yalavarthi S, et al. Neutrophil-mediated IFN activation in the bone marrow alters $B$ cell development in human and murine systemic lupus erythematosus. $J$ Immunol 2014;192:906-18

58. Hervier B, Beziat V, Haroche J, et al. Phenotype and function of natural killer cells in systemic lupus erythematosus: excess interferon- $\gamma$ production in patients with active disease. Arthritis Rheum 2011;63:1698-706.

59. Lin S-C, Kuo C-C, Tsao J-T, et al. Profiling the expression of interleukin (IL)-28 and IL-28 receptor $\alpha$ in systemic lupus erythematosus patients. Eur J Clin Invest 2012;42:61-9.

60. Hagberg $\mathrm{N}$, Berggren $\mathrm{O}$, Leonard $\mathrm{D}$, et al. IFN- $\alpha$ production by plasmacytoid dendritic cells stimulated with RNA-containing immune complexes is promoted by NK cells via MIP-1 $\beta$ and LFA-1. $\mathrm{J}$ Immunol 2011;186:5085-94.
61. Berggren O, Hagberg N, Weber G, et al. B lymphocytes enhance interferon- $\alpha$ production by plasmacytoid dendritic cells. Arthritis Rheum 2012;64:3409-19.

62. Leonard D, Eloranta M-L, Hagberg N, et al. Activated T cells enhance interferon- $\alpha$ production by plasmacytoid dendritic cells stimulated with RNA-containing immune complexes. Ann Rheum Dis 2016;75:1728-34.

63. Langefeld CD, Ainsworth HC, Cunninghame Graham DS, et al. Transancestral mapping and genetic load in systemic lupus erythematosus. Nat Commun 2017;8:16021.

64. Deng Y, Tsao BP. Updates in lupus genetics. Curr Rheumatol Rep 2017;19:68

65. Thorlacius GE, Wahren-Herlenius M, Rönnblom L. An update on the role of type I interferons in systemic lupus erythematosus and Sjögren's syndrome. Curr Opin Rheumatol 2018;30:471-81.

66. Remmers EF, Plenge RM, Lee AT, et al. STAT4 and the risk of rheumatoid arthritis and systemic lupus erythematosus. $N$ Engl $J$ Med 2007;357:977-86.

67. Svenungsson E, Gustafsson J, Leonard D, et al. A STAT4 risk allele is associated with ischaemic cerebrovascular events and antiphospholipid antibodies in systemic lupus erythematosus. Ann Rheum Dis 2010;69:834-40.

68. Bolin K, Sandling JK, Zickert A, et al. Association of STAT4 polymorphism with severe renal insufficiency in lupus nephritis. PLOS One 2013;8:e84450.

69. Taylor KE, Remmers EF, Lee AT, et al. Specificity of the STAT4 genetic association for severe disease manifestations of systemic lupus erythematosus. PLoS Genet 2008:4:e1000084.

70. Hagberg N, Joelsson M, Leonard D, et al. The STAT4 SLE risk allele rs $7574865 T$ is associated with increased IL-12-induced IFN- $\gamma$ production in T cells from patients with SLE. Ann Rheum Dis 2018;77:1070-7.

71. Hagberg N, Rönnblom L. Interferon- $\alpha$ enhances the IL-12-induced STAT4 activation selectively in carriers of the STAT4 SLE risk allele rs7574865[T]. Ann Rheum Dis 2019;78:429-31.

72. van Vollenhoven RF, Hahn BH, Tsokos GC, et al. Efficacy and safety of ustekinumab, an IL-12 and IL-23 inhibitor, in patients with active systemic lupus erythematosus: results of a multicentre, double-blind, phase 2, randomised, controlled study. Lancet 2018;392:1330-9.

73. Lee-Kirsch MA. The type I interferonopathies. Annu Rev Med 2017;68:297-315

74. Almlöf JC, Nystedt S, Leonard D, et al. Whole-genome sequencing identifies complex contributions to genetic risk by variants in genes causing monogenic systemic lupus erythematosus. Hum Genet 2019;138:141-50.

75. Lanata CM, Chung SA, Criswell LA. DNA methylation 101: what is important to know about DNA methylation and its role in SLE risk and disease heterogeneity. Lupus Sci Med 2018;5:e000285.

76. Imgenberg-Kreuz J, Carlsson Almlöf J, Leonard D, et al. DNA methylation mapping identifies gene regulatory effects in patients with systemic lupus erythematosus. Ann Rheum Dis 2018;77:736-43.

77. Ulff-Møller CJ, Asmar F, Liu Y, et al. Twin DNA methylation profiling reveals flare-dependent interferon signature and $B$ cell promoter hypermethylation in systemic lupus erythematosus. Arthritis Rheumatol 2018;70:878-90.

78. Attallah AM, Strong DM. Differential effects of interferon on the MHC expression of human lymphocytes. enhanced expression of HLA without effect on la. Int Arch Allergy Appl Immunol 1979;60:101-5.

79. CF Y, Peng WM, Oldenburg J, et al. Human plasmacytoid dendritic cells support Th17 cell effector function in response to TLR7 ligation. J Immunol 2010;184:1159-67.

80. Kiefer K, Oropallo MA, Cancro MP, et al. Role of type I interferons in the activation of autoreactive B cells. Immunol Cell Biol 2012;90:498-504.

81. Sjöstrand M, Johansson A, Aqrawi L, et al. The expression of BAFF is controlled by IRF transcription factors. J Immunol 2016;196:91-6.

82. Hagberg N, Theorell J, Hjorton K, et al. Functional anti-CD94/ NKG2A and anti-CD94/NKG2C autoantibodies in patients with systemic lupus erythematosus. Arthritis Rheumatol 2015;67:1000-11.

83. Biron CA, Nguyen KB, Pien GC, et al. Natural killer cells in antiviral defense: function and regulation by innate cytokines. Annu Rev Immunol 1999;17:189-220.

84. Thacker SG, Berthier CC, Mattinzoli D, et al. The detrimental effects of IFN- $\alpha$ on vasculogenesis in lupus are mediated by repression of IL-1 pathways: potential role in atherogenesis and renal vascular rarefaction. J Immunol 2010;185:4457-69. 
85. Denny MF, Thacker S, Mehta H, et al. Interferon-Alpha promotes abnormal vasculogenesis in lupus: a potential pathway for premature atherosclerosis. Blood 2007;110:2907-15.

86. Lee PY, Li Y, Richards HB, et al. Type I interferon as a novel risk factor for endothelial progenitor cell depletion and endothelial dysfunction in systemic lupus erythematosus. Arthritis Rheum 2007;56:3759-69.

87. Buie JJ, Renaud LL, Muise-Helmericks R, et al. IFN- $\alpha$ negatively regulates the expression of endothelial nitric oxide synthase and nitric oxide production: implications for systemic lupus erythematosus. J Immunol 2017;199:1979-88.

88. Li J, Fu Q, Cui H, et al. Interferon- $\alpha$ priming promotes lipid uptake and macrophage-derived foam cell formation: a novel link between interferon- $\alpha$ and atherosclerosis in lupus. Arthritis Rheum 2011;63:492-502.

89. Lood C, Amisten S, Gullstrand B, et al. Platelet transcriptional profile and protein expression in patients with systemic lupus erythematosus: up-regulation of the type I interferon system is strongly associated with vascular disease. Blood 2010;116:1951-7.

90. Liu Y, Kaplan MJ. Cardiovascular disease in systemic lupus erythematosus: an update. Curr Opin Rheumatol 2018;30:1-8.

91. Crow YJ, Lebon P, Casanova J-L, et al. A brief historical perspective on the pathological consequences of excessive type I interferon exposure in vivo. J Clin Immunol 2018;38:694-8.

92. Lazear HM, Schoggins JW, Diamond MS. Shared and distinct functions of type I and type III interferons. Immunity 2019:50:907-23.

93. Pollard KM, Cauvi DM, Toomey CB, et al. Interferon- $\gamma$ and systemic autoimmunity. Discov Med 2013;16:123-31.

94. Rönnblom L, Eloranta M-L, Alm GV. The type I interferon system in systemic lupus erythematosus. Arthritis Rheum 2006;54:408-20.

95. Peck-Radosavljevic M, Wichlas M, Homoncik-Kraml M, et al. Rapid suppression of hematopoiesis by standard or pegylated interferon- $\alpha$. Gastroenterology 2002;123:141-51.

96. Weckerle CE, Franek BS, Kelly JA, et al. Network analysis of associations between serum interferon- $\alpha$ activity, autoantibodies, and clinical features in systemic lupus erythematosus. Arthritis Rheum 2011;63:1044-53.

97. Jeremiah N, Neven B, Gentili M, et al. Inherited STING-activating mutation underlies a familial inflammatory syndrome with lupus-like manifestations. J Clin Invest 2014;124:5516-20.

98. Braunstein I, Klein R, Okawa J, et al. The interferon-regulated gene signature is elevated in subacute cutaneous lupus erythematosus and discoid lupus erythematosus and correlates with the cutaneous lupus area and severity index score. Br J Dermatol 2012;166:971-5.

99. Wenzel J, Wörenkämper E, Freutel S, et al. Enhanced type I interferon signalling promotes Th1-biased inflammation in cutaneous lupus erythematosus. J Pathol 2005;205:435-42.

100. Shipman WD, Chyou S, Ramanathan A, et al. A protective Langerhans cell-keratinocyte axis that is dysfunctional in photosensitivity. Sci Transl Med 2018;10:eaap9527.

101. Li TM, Shwartz N, Shipman WD, et al. Type I interferon modulates ADAM17 activity in photosensitive lupus mouse models. Lupus Sci Med 2019;13.

102. Merrill JT, Furie R, Werth VP, et al. Anifrolumab effects on rash and arthritis: impact of the type I interferon gene signature in the phase Ilb MUSE study in patients with systemic lupus erythematosus. Lupus Sci Med 2018;5:e000284.

103. Wallace DJ, Furie RA, Tanaka Y, et al. Baricitinib for systemic lupus erythematosus: a double-blind, randomised, placebo-controlled, phase 2 trial. Lancet 2018;392:222-31.

104. Sanchez GAM, Reinhardt A, Ramsey S, et al. JAK1/2 inhibition with baricitinib in the treatment of autoinflammatory interferonopathies. $J$ Clin Invest 2018;128:3041-52.
105. Toukap AN, Galant C, Theate I, et al. Identification of distinct gene expression profiles in the synovium of patients with systemic lupus erythematosus. Arthritis Rheum 2007;56:1579-88.

106. Catalina MD, Bachali P, Geraci NS, et al. Gene expression analysis delineates the potential roles of multiple interferons in systemic lupus erythematosus. Commun Biol 2019;2.

107. Higuchi A, Takanashi Y, Tsuzuki N, et al. Production of interferonbeta by fibroblast cells on membranes prepared with RGDcontaining peptides. J Biomed Mater Res A 2003;65:369-78.

108. Watanabe S, Imaizumi T, Tsuruga K, et al. Glomerular expression of myxovirus resistance protein 1 in human mesangial cells: possible activation of innate immunity in the pathogenesis of lupus nephritis. Nephrology 2013;18:833-7.

109. Castellano G, Cafiero C, Divella C, et al. Local synthesis of interferon-alpha in lupus nephritis is associated with type I interferons signature and LMP7 induction in renal tubular epithelial cells. Arthritis Res Ther 2015;17.

110. Peterson KS, Huang J-F, Zhu J, et al. Characterization of heterogeneity in the molecular pathogenesis of lupus nephritis from transcriptional profiles of laser-captured glomeruli. J Clin Invest 2004:113:1722-33.

111. Shimizu Y, Yasuda S, Kimura T, et al. Interferon-Inducible Mx1 protein is highly expressed in renal tissues from treatment-naïve lupus nephritis, but not in those under immunosuppressive treatment. Mod Rheumatol 2018;28:661-9.

112. Migliorini A, Angelotti ML, Mulay SR, et al. The antiviral cytokines IFN- $\alpha$ and IFN- $\beta$ modulate parietal epithelial cells and promote podocyte loss: implications for IFN toxicity, viral glomerulonephritis, and glomerular regeneration. Am J Pathol 2013;183:431-40.

113. Lebon $P$, Lenoir GR, Fischer $A$, et al. Synthesis of intrathecal interferon in systemic lupus erythematosus with neurological complications. Br Med J 1983;287:1165-7.

114. Shiozawa S, Kuroki Y, Kim M, et al. Interferon-alpha in lupus psychosis. Arthritis Rheum 1992;35:417-22.

115. Raison CL, Borisov AS, Broadwell SD, et al. Depression during pegylated interferon-alpha plus ribavirin therapy: prevalence and prediction. J Clin Psychiatry 2005;66:41-8.

116. Santer DM, Yoshio T, Minota S, et al. Potent induction of IFN- $\alpha$ and chemokines by autoantibodies in the cerebrospinal fluid of patients with neuropsychiatric lupus. J Immunol 2009;182:1192-201.

117. Bialas AR, Presumey J, Das A, et al. Microglia-dependent synapse loss in type I interferon-mediated lupus. Nature 2017;546:539-43.

118. Felten R, Dervovic E, Chasset F, et al. The 2018 pipeline of targeted therapies under clinical development for systemic lupus erythematosus: a systematic review of trials. Autoimmun Rev 2018;17:781-90.

119. Baccala R, Gonzalez-Quintial R, Schreiber RD, et al. Anti-IFN$\alpha / \beta$ receptor antibody treatment ameliorates disease in lupuspredisposed mice. J Immunol 2012;189:5976-84.

120. Munroe ME, Lu R, Zhao YD, et al. Altered type II interferon precedes autoantibody accrual and elevated type I interferon activity prior to systemic lupus erythematosus classification. Ann Rheum Dis 2016:75:2014-21.

121. El-Sherbiny YM, Psarras A, Md Yusof MY, et al. Publisher correction: A novel two-score system for interferon status segregates autoimmune diseases and correlates with clinical features. Sci Rep 2018;8:14846.

122. Der E, Suryawanshi H, Morozov P, et al. Tubular cell and keratinocyte single-cell transcriptomics applied to lupus nephritis reveal type I IFN and fibrosis relevant pathways. Nat Immunol 2019:20:915-27.

123. Reid $\mathrm{S}$, Alexsson $\mathrm{A}$, Frodlund $\mathrm{M}$, et al. A high genetic risk score is associated with early disease onset, organ damage and decreased survival in systemic lupus erythematosus. Lupus Sci Med 2019;6:A154-5. 